\title{
A Novel Approach for Dynamic Polarity Mining from Customer Reviews
}

\author{
Yuanchao Liu ${ }^{1}$, Xin Wang ${ }^{1}$, Chengjie $\operatorname{Sun}^{1} \&$ Bingquan Liu ${ }^{1}$ \\ ${ }^{1}$ School of Computer Science and Technology, Harbin Institute of Technology, Harbin, China \\ Correspondence: Yuanchao Liu, School of Computer Science and Technology, Harbin Institute of Technology, \\ Harbin, China. E-mail: lyc@insun.hit.edu.cn
}

\author{
Received: April 12, 2013 Accepted: May 17, 2013 Online Published: June 28, 2013 \\ doi:10.5539/cis.v6n3p80 URL: http://dx.doi.org/10.5539/cis.v6n3p80
}

This research was supported by "the Fundamental Research Funds for the Central Universities" (Grant No.HIT.NSRIF.2009065) and Key Laboratory Opening Funding of China MOE-MS Key Laboratory of Natural Language Processing and Speech (HIT.KLOF.2009022)

\begin{abstract}
The dynamic opinion words usually have different polarity directions when they are in combination with different features. Determining the polarity direction of these dynamic opinion words is one of the difficult problems in opinion mining. Although the opinion words with dynamic polarity are usually less than those with static polarity, these opinion words can be matched with most features, can appear very frequently in customer reviews. So the impact on the overall feature-opinion extraction accuracy and the calculation of comprehensive consumer word of mouth cannot be ignored. In this paper, we address this issue of judging the polarity direction of dynamic opinion words in different feature contexts by means of customer review mining and voting strategy. Our approach is based on this hypothesis: when the corpus scale is big enough, the word of mouth of product features are relatively stable. The experimental results verified the effectiveness of our method. Although the test is performed in mobile phone review areas, the approach can be easily applied to other areas.
\end{abstract}

Keywords: dynamic polarity, polarity identification, sentiment analysis, customer review mining

\section{Introduction}

With the continuous increase of Internet, there emerge many customer reviews on the on-line e-commerce website and online forums (Liu \& Yong, 2010; Chen, 2010). Customer review is valuable for both manufacturers and consumers: Manufacturers can analyze the information to obtain feedback and comments; consumers can read others' review to help their purchases decision. However, the artificial collecting-reading-analyzing cycle of such kinds of information from websites has become very cumbersome and time-consuming. In term of this background, Opinion mining and quantity analysis of product review have become more important (Prabhudev, 2005; Liu, 2011).

Product opinion mining is a very meaningful technology to analyze and mine the valuable knowledge hidden in the reviews expressed by past customers. One of the important research directions is to mine out the feature-opinion pair from customer review sentences. In such research, many opinion words with polarity direction will be inevitability used in such reviews. In fact, the research of opinion word polarity analysis is the basis of many Product opinion mining and sentiment analysis work. There exist many opinion words which polarity direction can be known and confirmed in any circumstances $(\mathrm{Lu}, 2009 ; \mathrm{Li}, 2010)$, and these words are usually called static polarity words, and are usually stored beforehand and loaded when performing sentient analysis. For example, the polarity words including “漂亮 (beautiful)”, “优雅 (graceful)”, “丑陃 (ugly)” have stable polarity value in almost any circumstance, we call these words stable polarity words.

There are also some opinion words which have dynamic polarity direction. The polarity of such words cannot be determined beforehand, as their direction change in different context. The polarities of such words are not stable, and usually vary when matched with different features. For example, the words “大 (big)”, “小 (small)”, “高 (high)”, “低 (low)”, “多 (more)”, “少 (less)” are dynamic polarity word. Although compared with static polarity word, the number of such words may be not so much, but most features can be matched with these 
polarity words, and these words are also very frequent. So the impact on sentiment mining can not be neglected. And it is necessary to determine the polarity value when matched with different features.

In this paper, we address this issue of judging the polarity direction of dynamic opinion words in different feature contexts by means of customer review mining and voting strategy. Our approach is based on this hypothesis: When the corpus scale is big enough, the word of mouth of product features are relatively stable.

The remaining content of this paper is organized as follows. Section 2 introduces related work. Section 3 describes the objective of our work. Section 4 proposes the dynamic polarity mining method. Section 5 presents the experimental results and analysis. In the end we draw conclusions and discuss some potential extensions of our approach in Section 6.

\section{Related Works}

Various approaches have been proposed in polarity discovery technology (Xu, 2011; Aurangzeb, 2011). Kim and Hovy (2004) use the relation of synonyms and antonyms in WordNet to determine the polarity of new terms which are associated with these words. However, their work need manual annotation, and the polarity term strength cannot be determined. Nozomi Kobayashi (2001) proposed a bootstrapping way to identify the semantic direction of opinion words. The problem of distinguishing semantic direction is converted to text sentiment classification problem. They used 52 hand-constructed rules for emotional classification of the sentence. Xi-wen Fang et al. (2008) use HowNet and the Harvard\&Lasswell dictionaries to get the static polarity, they make statistics on the dependence relation in the emotional corpus to construct the dynamically polarity word Dictionary. But the size of dynamic polarity dictionary is also limited because the corpus has limited size.

Cai Jianping and Shi-ping Lin (2007) build Chinese polarity Dictionary SentiHowNet based on the conversion of English polar SentiWordnet and Hownet. Their Experimental results show that recall and precision rates are not high, the main factors affecting the recall rate is that some terms have more than meaning. And the terms collected are not very comprehensive. Such obstacle may be overcome by building domain ontology in order to obtain higher recall.

There are also some other research topics in this field. Kim (2004) focuses on identifying subjective expressions or recognizing contextual polarity in phrase-level sentiment analysis. The research on Sentiment analysis using subjectivity summarization based on minimum cuts has been taken by Pang and Lee (2004). Deeper sentiment analysis using machine translation technology is also performed by Hiroshi, Tetsuya, and Hideo (2004). These studies are actually about upper and deeper emotional information mining.

\section{The Objective of Our Work}

Table 1 is the format of static polarity word. Such knowledge can usually be obtained from some lexicon such as HowNet. The format of dynamic polarity word is shown in Table 2. It adds a context feature column compared with table 1, as these words will usually have different polarity direction when matched with different feature. For example, the polarity of “大 (big)” is negative when matched with “噪音 (noise)” and is positive when matched with “内存 (memory)". In addition there is also a need to consider the problem of synonym problem. For example, the polarity of opinion word “大 (big)” is negative when matched with another feature “噪声 (noise)”, which is a synonym of “噪音 (noise)”. whereas the polarity of “大 (big)” is positive when matched with “价值 (value)". So the objective this paper is to find the direction of dynamic polarity words in different context, as shown in Table 2.

Table 1. Format of static polarity opinion word

\begin{tabular}{clccc}
\hline NO & Opinion word & Pol. direction & Strength & Examples \\
\hline 1 & 漂亮 (beautiful) & + & 1.0 & 外观非常漂亮 (its appearance is very beautiful) \\
2 & 难看 (ugly) & - & 1.0 & 外形比较难看 (the screen of this model is ugly) \\
$\ldots . .$. & $\ldots . .$. & $\ldots .$. & $\ldots .$. & $\ldots .$. \\
\hline
\end{tabular}


Table 2. Format of dynamic polarity opinion word

\begin{tabular}{cccccc}
\hline NO & Opinion word & Feature & Pol. direction & Strength & Examples \\
\hline 1 & 大 (big) & 噪音 (noise) & + & 1.0 & 噪声太大 (the noise of is too big) \\
2 & 大 (big) & 价值 (value) & - & 1.0 & 手机的使用价值大 (this model has big value) \\
& $\ldots . .$. & & $\ldots .$. & $\ldots .$. & $\ldots .$. \\
\hline
\end{tabular}

\section{Dynamic Polarity Mining}

Although we can also label the direction of dynamic polarity words in different context as many other static dictionaries, such work will consume more human labor. In this paper, by analyzing the actual customer review context, we proposed a semi-automated method to determine the direction of dynamic polarity word.

We first make some definitions here:

Comprehensive word of mouth CWM (P, Pm, F): P denote the type of some products, e.g. P = "mobile phone"; $\mathrm{Pm}$ denote the product model, e.g. $\mathrm{Pm}=$ "Philips 9100"; F denote one product feature, e.g. $\mathrm{F}=$ "screen". CWM (P, Pm, F) defines the overall market reputation for parameter (P, Pm, F), its possible value is (POS, NEG), where POS means “+”, NEG means “".".

Two assumptions are presented here:

Assumption 1: when the customer review corpus is big enough, the comprehensive word of mouth CWM (P, Pm, F) for the feature $\mathrm{F}$ of product model $\mathrm{Pm}$ is stable.

The proof is as follows. CWM (P, Pm, F) means the polarity direction of most users for feature $\mathrm{F}$ and product model Pm. Take the feature ”耗电量 (power consummation)" of mobile phone ( $\mathrm{P}=$ “手机 (mobile phone)”, $\mathrm{F}=$ “耗电量 (power consummation)”) as a example, even though the comprehensive polarity value of different product model may be different, but for some particular product model Pm, in most situations, the comprehensive review can be stable. For example, the polarity of feature F “耗电量” (“power consummation”) for Pm "Philips 9100" is positive. Here the calculation of the CWM (P, Pm, F) can utilize the results of feature-pair extraction, which utilize the static polarity lexicon.

Assumption 2: for product $\mathrm{P}$, product model $\mathrm{Pm}$ and feature $\mathrm{F}$, if there are two corresponding dynamic polarity words DPW1, DPW2 which can both match with feature F and have opposite direction with each other (i.e., they are antonyms). When the customer review corpus is big enough, the frequency of one dynamic polarity word will usually be greater than that of another.

For example, for triple ( $\mathrm{P}=$ “mobile phone"; $\mathrm{Pm}$ = “the Philips 9100”; $\mathrm{F}$ = “耗电量 (power consummation)"), DPW1 = “大 (more)”, DPW2 = “小 (less)”. It can be found obviously that the comments which says “耗电量 (power consummation)” “小 (less)” are more frequent than the comments which says “耗电量 (power consummation)" “大 (more)".

For the above example, if most reviews are positive, then the comprehensive value CWM (P, Pm, F) will be positive. There are 2 problems to be considered for these two assumptions: 1) the reviews for some type of product are rare, i.e. the corpus may be not so big enough; 2) the reviews for some type of product are not clear, i.e. the positive number may be near to the negative number, thus it is not easy to calculate CWM (P, Pm, F). To deal with these problems, many product models are analyzed and a voting mechanism is adopted in this paper.

According to the above definitions and assumptions, the method of determining the dynamic polarity is shown in algorithm 1 . 


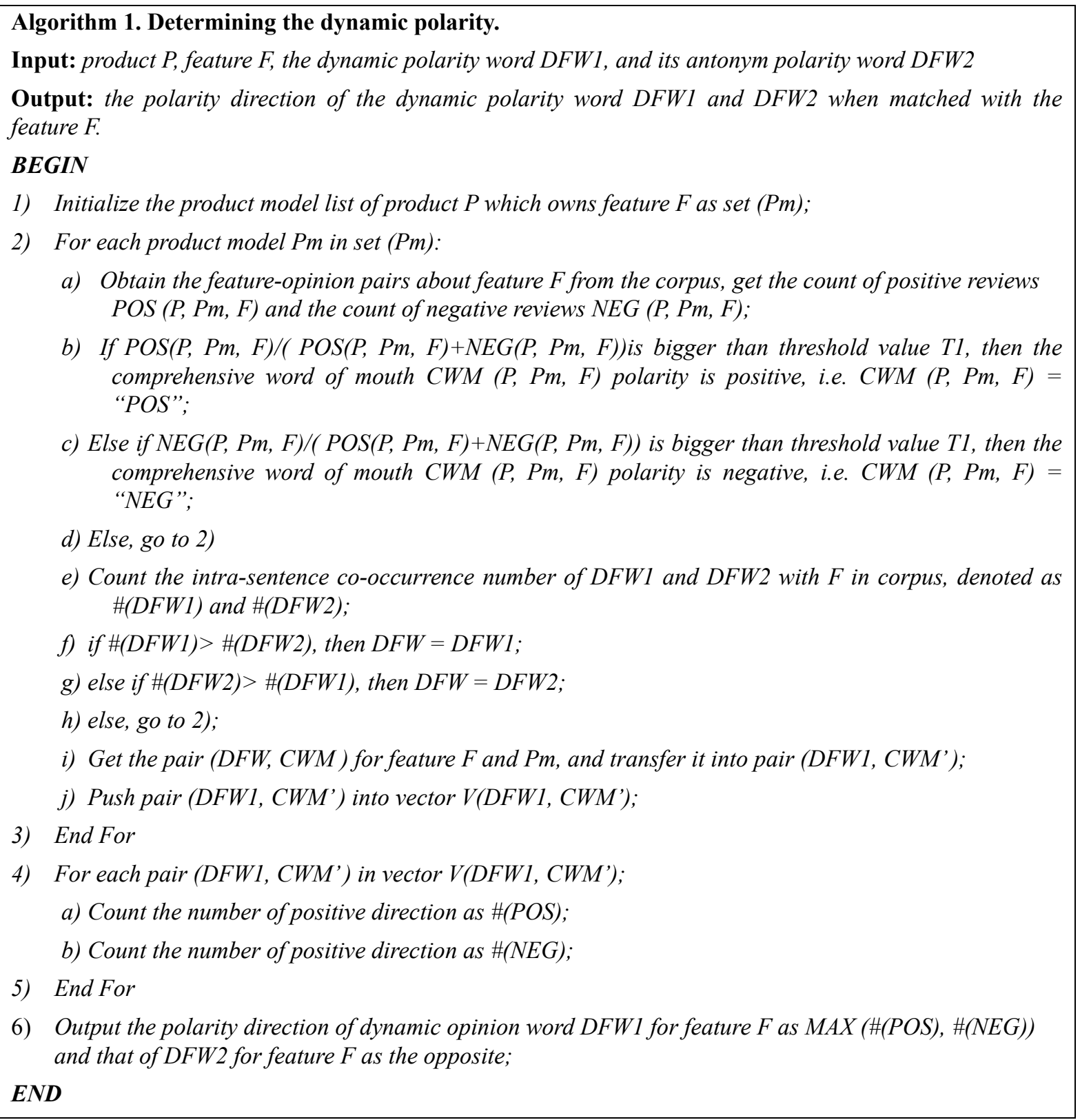

In algorithm 1, the first thing is to determine the comprehensive reputation of the product $\mathrm{P}$ on feature $\mathrm{F}$, i.e. CWM (P, Pm, F). Calculation of CWM (P, Pm, F) is performed by making use of the extraction results of feature-opinion pairs, which is based on Heuristic rules and static polarity dictionary. For the features of some product models, the review corpus may be rare, or the Comprehensive reputation is not clear. So for same feature, many product models are used to determine the Comprehensive reputation. The second step is to count and select the dynamic polarity word which has more frequent co-occurrence with feature $\mathrm{F}$ in corpus. The last step is to determine the polarity direction of the dynamic polarity word DFW when matched with feature $\mathrm{F}$ by using voting mechanism.

The step 2) $\rightarrow$ a) in algorithm 1 needs the help of feature-opinion pair extraction, we use the approach in Song (2011) to achieve this task. The basic idea is: a combination of 2 kinds of knowledge for feature-opinion extraction: statistical knowledge and grammatical knowledge. The extraction is opinion-word-driven approach, as the opinion word in sentence usually means there are features in its context. The statistical knowledge is based on the observation that there are usually some more stable co-occurrence for some features and opinion words. The grammatical approach is based the observation that there exists some grammatical patterns between feature 
and opinion. These patterns include both POS rules and dependency relations rules. There are 21 POS rules. For example, one POS rule is “n ad d a” with the example ”外观/n 确实/ad 很/d 漂亮/a". There are 5 dependency relations rules. For example, one dependency relations rule is "SBV". The corresponding example is shown in Figure 1, as the relation between feature “外观 (appearance)" and opinion “漂亮 (beautiful)" is “SBV”.

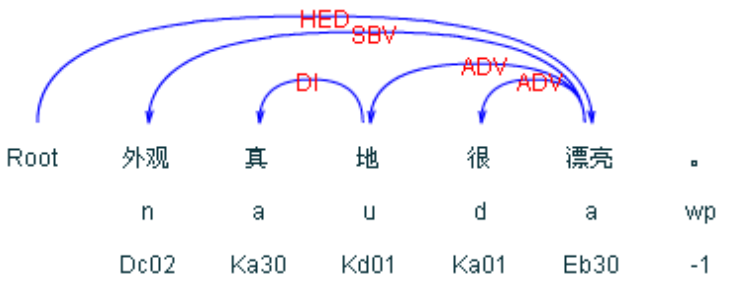

Figure 1. One dependency relations example

The basic principle can also be shown by an example in Figure 2. As shown in Figure 2, the frequency of “小 (small)" is three and bigger than that of "大 (big)", which frequency is one. In feature-opinion extraction results of the four product models, the number of negative polarity is bigger than that of positive (3:1), so the dominant polarity direction is negative and the dominant dynamic polarity word is “小 (small)", thus the value of dynamic polarity word "小 (small)" is negative when matched with feature F. correspondingly, the value of dynamic polarity word "大 (big)" will be positive when matched with feature F.

\begin{tabular}{|c|c|c|c|c|c|c|}
\hline \multicolumn{6}{|c|}{ The condition attribute } & \multirow{2}{*}{$\begin{array}{l}\text { The decision } \\
\text { The polarity direction } \\
\text { for DFW1 }\end{array}$} \\
\hline $\begin{array}{c}\text { Dynamic polarity } \\
\text { word }\end{array}$ & Feature & Product & $\mathrm{Pm}$ & $\begin{array}{c}\text { Dominant dynamic } \\
\text { polarity word }\end{array}$ & $\begin{array}{c}\text { Dominant polarity } \\
\text { direction }\end{array}$ & \\
\hline \multirow{4}{*}{$\begin{array}{c}\text { DFW1="大(big)" } \\
\text { DFW2="小(small)" }\end{array}$} & \multirow{4}{*}{$\begin{array}{l}F=" \text { 屏萧 } \\
\text { (screen)" }\end{array}$} & \multirow{4}{*}{$\begin{array}{l}P=\text { "mobil } \\
\text { e phone" }\end{array}$} & PM1 & 大(big) & POS & \multirow{4}{*}{ 小(small) $->N E G$} \\
\hline & & & $\mathrm{PM} 2$ & 小(small) & NEG & \\
\hline & & & $\mathrm{PM} 3$ & 小(small) & NEG & \\
\hline & & & PM4 & 小 (small) & NEG & \\
\hline
\end{tabular}

Figure 2. An example of determining the direction of dynamic polarity word

\section{Experimental Results and Analysis}

The purpose of the experiment is to test the effects of the method in this research. We tested our approach on mobile phone reviews, which are downloaded from two famous e-commerce websites (http://pinglun.it168.com; http://mobile.zol.com.cn/), and there are 1729 reviews used for performance evaluation. The F-O pair frequency used in this paper has been counted beforehand on 16M customer review corpus and 96745 F-O Pairs are counted. In Table 3, we shows the product models and their ID, and in Table 4, Some top-frequent features which co-occur with dynamic opinion words are demonstrated, for example “屏幕 (screen)”, “字体 (font)”, “键盘 (keyboard)" and etc.

The evaluation method is as follows: assuming there are totally $\mathrm{C}$ feature $\&$ dynamic word pairs, and the polarity of C' pairs are found correctly, and then the accuracy rate can be calculated as follows:

$$
\text { Accuracy Rate }=C^{\prime} / C
$$

Table 3. The product models and their IDs

\begin{tabular}{clclclll}
\hline ID & Product Model & ID & Product Model & ID & Product Model & ID & Product Model \\
\hline 1 & Nokia N95 & 7 & Samsung I8000 & 13 & Blackberry 8310 & 19 & HTC Incredible S \\
2 & Nokia N96 & 8 & Samsung I900 & 14 & Blackberry 9780 & 20 & LG GD580 \\
3 & Nokia N81 & 9 & Moto ME722 & 15 & HTC Desire & 21 & LG GT540 \\
4 & Samsung I9000 & 10 & Moto ME600 & 16 & HTC Legend & 22 & LG BL20e \\
5 & Samsung I5700 & 11 & Moto XT702 & 17 & HTC Hero & 23 & Apple iPhone 4 \\
6 & Samsung T959 & 12 & Moto XT800 & 18 & HTC HD2 & 24 & Apple iPhone 3GS \\
\hline
\end{tabular}


Table 4. Some top-frequent features which co-occur with dynamic opinion words

\begin{tabular}{lll}
\hline NO. & Feature & Dynamic polarity word \\
\hline 1 & 屏幕 (screen) & 大小 (big or small) \\
2 & 字体(font) & 大小 (big or small) \\
3 & 键盘(keyboard) & 大小 (big or small) \\
4 & 程序(program) & 多少(more or less) \\
5 & 内存(memory) & 大小 (big or small) \\
6 & 配置(setting) & 高低(high or low) \\
7 & 待机时间(standby time) & 长短(long or short) \\
8 & 通话时间(communication time) & 长短(long or short) \\
9 & 功能(function) & 多少(more or less) \\
10 & 应用程序(application) & 多少(more or less) \\
11 & 软件(software) & 多少(more or less) \\
12 & 声音(speech) & 大小 (big or small) \\
13 & 性价比(performance price ratio) & 高低(high or low) \\
14 & 性能(performance) & 高低(high or low) \\
15 & 质量(quality) & 高低(high or low) \\
16 & 容量(capacity) & 高低(high or low) \\
17 & 音质(voice quality) & 高低(high or low) \\
18 & 亮度(brightness) & 高低(high or low) \\
19 & 分辨率(resolution) & 高低(high or low) \\
\hline & 价格(price) & 高低(high or low) \\
\hline & &
\end{tabular}

Table 5 examines the process of determining the direction of dynamic polarity word by using many product models, and the meaning of each row is explained as the follows. The feature is "standby time" and dynamic polarity word is "long" or "short". The "dominant static polarity" row for each product model is obtained by extracting Feature-opinion pairs, as briefly introduced in section 4. For example, if the "dominant static polarity" is "NEG", it will mean that the frequency of "NEG" is bigger than that of "POS". Column 2 means the number of positive sentences divided by the sum of positive sentences and negative sentences. If that value is bigger than 0.5 , the "dominant static polarity" will be negative. The "dominant dynamic polarity" is the dynamic polarity word which has bigger frequency than that with other direction. For example, if the dominant dynamic polarity is "SHORT", then it means the frequency of "SHORT" is bigger than that of "LONG". In this way, Conclusion 1 and Conclusion 2 in Table 5 are drawn correspondingly.

It can be seen from Table 5 that: 1) basically, many dominant static polarities are negative, as users tend to complain about the products they bought. 2) Most rows in table 5 are correct, and there are 4 out of 24 conclusions are not correct (which has been underlined). Basically, the overall conclusion can be obtained from Table 5: when "long" is matched with "standby time" the direction is "POS".

In Figure 3, the accuracy comparison of dynamic polarity word mining is demonstrated (the X-axis includes the features shown in Table 4). The baseline method is as follows: assuming the dominant direction is negative as it can be found that users tend to make negative review on products. Recognition results using this method are as the baseline. It is shown in Figure 3 that, our approach outperforms that baseline method in most cases. 
Table 5. Determining the direction of dynamic polarity word by using many product models (feature: "standby time"; dynamic polarity word: long or short)

\begin{tabular}{|c|c|c|c|c|c|}
\hline ID & $\begin{array}{l}\text { \# of pos./(\# of pos. } \\
\text { +\# of neg.) }\end{array}$ & $\begin{array}{l}\text { Dominant static } \\
\text { polarity }\end{array}$ & $\begin{array}{l}\text { Dominant dynamic } \\
\text { polarity }\end{array}$ & Conclusion 1 & Conclusion 2 \\
\hline 1 & 0.1875 & NEG & SHORT & standby time-short-NEG & standby time-long-POS \\
\hline 2 & 0.2143 & NEG & SHORT & standby time-short-NEG & standby time-long-POS \\
\hline 3 & 0.3500 & NEG & LONG & standby time-long-NEG & standby time-long-NEG \\
\hline 4 & 0.2162 & NEG & SHORT & standby time-short-NEG & standby time-long-POS \\
\hline 5 & 0.1250 & NEG & SHORT & standby time-short-NEG & standby time-long-POS \\
\hline 6 & 0.3333 & NEG & SHORT & standby time-short-NEG & standby time-long-POS \\
\hline 7 & 0.8500 & POS & LONG & standby time-long-POS & standby time-long-POS \\
\hline 8 & 0.4286 & NEG & SHORT & standby time-short-NEG & standby time-long-POS \\
\hline 9 & 0.6000 & POS & LONG & standby time-long-POS & standby time-long-POS \\
\hline 10 & 0.1429 & NEG & SHORT & standby time-short-NEG & standby time-long-POS \\
\hline 11 & 0.0909 & NEG & LONG & $\underline{\text { standbv time-long-NEG }}$ & $\underline{\text { standby time-long-NEG }}$ \\
\hline 12 & 0.2727 & NEG & LONG & $\underline{\text { standby time-long-NEG }}$ & $\underline{\text { standby time-long-NEG }}$ \\
\hline 13 & 0.4286 & NEG & SHORT & standby time-short-NEG & standby time-long-POS \\
\hline 14 & 0.6667 & POS & SHORT & standby time-short-NEG & standby time-long-POS \\
\hline 15 & 0.3333 & NEG & SHORT & standby time-short-NEG & standby time-long-POS \\
\hline 16 & 0.2941 & NEG & SHORT & standby time-short-NEG & standby time-long-POS \\
\hline 17 & 0.1429 & NEG & SHORT & standby time-short-NEG & standby time-long-POS \\
\hline 18 & 0.4615 & NEG & SHORT & standby time-short-NEG & standby time-long-POS \\
\hline 19 & 0.2045 & NEG & SHORT & standby time-short-NEG & standby time-long-POS \\
\hline 20 & 0.6666 & POS & SHORT & standbv time-short-POS & standby time-short-POS \\
\hline 21 & 0.6666 & POS & LONG & standby time-long-POS & standby time-long-POS \\
\hline 22 & 0.0769 & NEG & SHORT & standby time-short-NEG & standby time-long-POS \\
\hline 23 & 0.2857 & NEG & SHORT & standby time-short-NEG & standby time-long-POS \\
\hline 24 & 0.4232 & NEG & SHORT & standby time-short-NEG & standby time-long-POS \\
\hline
\end{tabular}

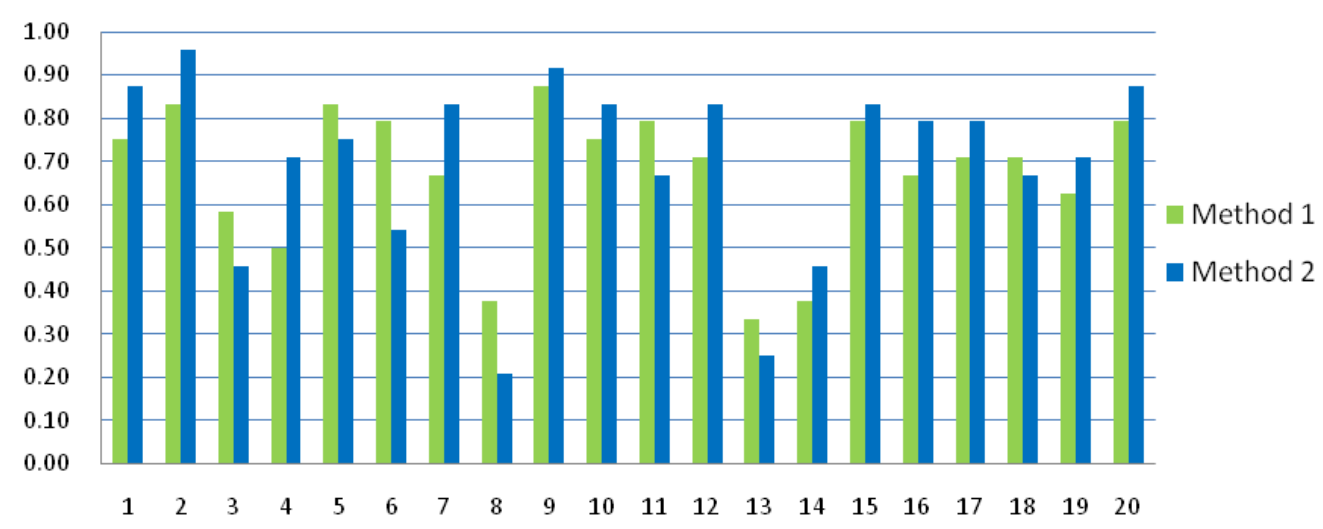

Figure 3. The accuracy comparison of dynamic polarity word mining on different features (X-axis: features, Y-axis: accuracy) 
It is noted that there are in fact many common characteristics for many electronic products and the sentiment standard is also very near. For example, for some electronic products which have photo function such as mobile phone and digital camera, “焦距 (focus)" “大 (big)” is positive review. So our approach can be easily transferred and applied in other products besides from mobile phone.

Besides, although in most cases, the sentiment is usually positive when the features are matched with dynamic words such as "big, "multiple", "long" and so on, and the sentiment is negative when matched with "small", "less", "short" and etc. A more notable exception is that, there are some features which have their own polar characteristics. such as “噪声 (noise)”, “故障 (error)”, “毛病 (problem)” and so on. For such words, the situation is clearly the opposite. These features usually have negative polarity when matched with some polarity of a greater degree and have positive polarity when matched with some polarity of a smaller degree. Thus the proposed method may achieve better performance on many features than the baseline method.

\section{Conclusions}

In this paper, we address the issue of dynamic polarity mining by means of voting strategy on the statistical analysis of static feature-opinion mining. The experimental results show that it is feasible for determining the direction of dynamic polarity. In theory, our approach can be easily applied to other areas. besides, our work can also help identify the so-called "negative” features, such as “噪音 (noise)”, “耗电量 (power consummation)”. The knowledge base obtained can provide stable help and support for high-precision products review mining.

As for future work, there is still need for further in-depth analysis from a deep-level perspective. The performance can be improved furthermore by designing a better way to improve the static feature-opinion mining performance, which may help improving the identification accuracy dynamic polarity word. Another problem to be considered in future to deal with the features with same or near meaning which are matched with dynamic opinion word, e.g. the polarity value of “大 (big)" matched with “噪声 (noise)" will be same when it is matched with “噪音 (noise)".

\section{References}

Aurangzeb, K., Baharum, B., \& Khairullah, K. (2011). Sentiment Classification Using Sentence-level Lexical Based Semantic Orientation of Online Reviews. Trends in Applied Sciences Research, 6, 1141-1157. http://dx.doi.org/10.3923/tasr.2011.1141.1157

Cai, J., \& Lin, S. (2007). Polarity analysis of lexicon and sentence based on machine learning. The third Chinese conference for information retrieval and content security (pp. 643-649). Suzhou, China.

Chen, H. (2010). Business and market intelligence 2.0. IEEE Intelligent Systems, 25(1), 68-71. http://dx.doi.org/10.1109/MIS.2010.27

Fang, X., \& Yao, T. (2008). Design of Chinese polarity lexicon. Seminar of Hownet, 38-46.

Hiroshi, K., Tetsuya, N., \& Hideo, W. (2004). Deeper sentiment analysis using machine translation technology. Proceedings of the 20th international conference on computational linguistics (COLING 2004) Geneva, Switzerland (pp. 494-500). http://dx.doi.org/10.3115/1220355.1220426

Kim, S. M., \& Hovy, E. (2004a). Determining the Sentiment of Opinions. Proceedings of the 20th international conference on computational linguistics (COLING 2004) (pp. 1367-1373). Geneva, Switzerland. http://dx.doi.org/10.3115/1220355.1220555

Kim, S. M., \& Hovy, E. (2004b). Determining the Sentiment of Opinions. Proceedings of the COLING conference, Geneva.

Kobayashi, N., Inui, T., \& Inui, K. (2001). Dictionary-based acquisition of the lexical knowledge for $\mathrm{p} / \mathrm{n}$ analysis (in Japanese). In Proceedings of Japanese Society for Artificial Intelligence (pp. 45-50).

Li, F., Han, C., Huang, M. et al. (2010). Structure-Aware Review Mining and Summarization. The 23rd International Conference on Computational Linguistics (COLING 2010) (pp. 653-661). Beijing.

Liu, Y., Chen, Y., Robert, F. L., Chen, H., Zimbra, D., \& Zeng, S. (2010). User-generated content on social media: Predicting market success with online word-of-mouth. IEEE Intelligent Systems, 25(1), 75-78.

Liu, Y., Cheng, H. K., Tang, Q. C., \& Enes, E. (2011). Optimal software pricing in the presence of piracy and $\begin{array}{lllll}\text { word-of-mouth } \quad \text { effect. Decision } & \text { Support }\end{array}$ http://dx.doi.org/10.1016/j.dss.2010.11.032 
Lu, Y., Zhai, C., \& Sundaresan, N. (2009). Rated aspect summarization of short comments. Proceedings of the 18th international conference on World wide web (WWW'09) (pp. 131-140). Madrid, Spain. http://dx.doi.org/10.1145/1526709.1526728

Pang, B., \& Lee, L. (2004). A sentimental education: Sentiment analysis using subjectivity summarization based on minimum cuts. In Proceedings of the 42nd annual meeting of the Association for Computational Linguistics Barcelona, Spain (pp. 271-278).

Prabhudev, K., \& Sridhar, B. (2005). The Social-Economic-Psychological model of technology adoption and usage: an application to online investing. Decision Support Systems, 39(3), 505-524. http://dx.doi.org/10.1016/j.dss.2003.12.003

Song, M. (2011). Research on Key Technologies of Quantitative of Internet Product Reviews. Master's thesis. Harbin institute of technology (pp. 15-36).

Xu, K. Q., Liao, S. Y., Li, J. X., \& Song, Y. X. (2011). Mining comparative opinions from customer reviews for Competitive Intelligence. Decision Support $\quad$ Systems, $50(4), \quad 734$. http://dx.doi.org/10.1016/j.dss.2010.08.021

\section{Copyrights}

Copyright for this article is retained by the author(s), with first publication rights granted to the journal.

This is an open-access article distributed under the terms and conditions of the Creative Commons Attribution license (http://creativecommons.org/licenses/by/3.0/). 